\title{
Eosinophilic gastroenteritis and related eosinophilic disorders
}

\author{
Calman Prussin, M.D. \\ Laboratory of Allergic Diseases, National Institute of Allergy and Infectious Diseases, National \\ Institutes of Health
}

\begin{abstract}
Eosinophilic gastroenteritis (EGE) represents one member within the spectrum of diseases collectively referred to as eosinophilic gastrointestinal disorders (EGIDs), which includes eosinophilic esophagitis (EoE), gastritis, enteritis, and colitis. EGE is less common than EoE and involves a different site of disease, but otherwise shares many common features with EoE. The clinical manifestations of EGE are protean and can vary from nausea and vomiting to protein losing enteropathy or even bowel obstruction requiring surgery. Although systemic corticosteroids are an effective treatment for EGE, their use over the chronic course of the disease results in substantial corticosteroid toxicity. Accordingly, there is a great need for improved therapies for these patients.
\end{abstract}

\section{Keywords}

Eosinophilic gastroenteritis; Eosinophilic gastritis; Eosinophilia; Food allergy; EGID

\section{Clinical presentation of EGE}

Eosinophilic gastroenteritis (EGE) represents one member within the spectrum of diseases collectively referred to as eosinophilic gastrointestinal disorders (EGIDs), which includes eosinophilic esophagitis (EoE), gastritis, enteritis, and colitis. Although some patients present with EGID limited to the stomach (eosinophilic gastritis, EG) or duodenum (eosinophilic duodenitis, ED), it is often simplest to refer to the combined entity of EGE. EoE as a clinical entity is effectively limited to "solitary EoE"; patients having coexistent EoE and EGE are a small minority. EoE and EGE are closely related disease entities, the relationship of which is discussed below. The diagnosis of EG is confirmed by a characteristic biopsy and/or eosinophilic ascitic fluid in the absence of other causes of gut eosinophilia.

10 Center Drive, MSC-1881, Bethesda, MD, 20892-1881, 301-496-1306, cprussin@niaid.nih.gov.

Publisher's Disclaimer: This is a PDF file of an unedited manuscript that has been accepted for publication. As a service to our customers we are providing this early version of the manuscript. The manuscript will undergo copyediting, typesetting, and review of the resulting proof before it is published in its final citable form. Please note that during the production process errors may be discovered which could affect the content, and all legal disclaimers that apply to the journal pertain.

Disclosures:

The author has no competing financial interests. 
The disease can affect patients of any age, but case series have noted a dominance of presentations starting in the third through fifth decade. As the prevalence of EoE has increased and there is an overall greater appreciation of EGID, it is likely that a second peak of incidence in the first decade of life will become better appreciated. As with EoE, there is a clear male predominance. An electronic survey sent to North American Allergists and Pediatric Gastroenterologists indicate prevalence for EGE of 22-28 per 100,000 persons ${ }^{1}$. Although no large longitudinal study has been performed, EGE is largely understood to be a chronic disease with few remissions after the first year.

The clinical features of EGE are protean and are related to the organs, tissue layers affected, and the intensity of eosinophilic inflammation ${ }^{2-6}$. Some patients present with dominant gastric or duodenal disease, whereas others have involvement of both organs. Dominant gastric disease often presents with nausea, vomiting and early satiety. In contrast, dominant duodenal disease may present with malabsorption and protein losing enteropathy. Both forms of EGE often have crampy abdominal pain and bloating as additional features. Because jejunal and ileal biopsies are not routinely obtained on endoscopy, it is not known how much these gut segments contribute to disease. Patients can variably present with either diarrhea or constipation ${ }^{7}$.

In addition to the varying distribution of eosinophils along the length of the GI tract, multiple reports have cited EGE subtypes based on differing depth of eosinophilic infiltration ${ }^{3,8}$. The 3 well-described subtypes include dominant involvement of the mucosal, muscularis, and subserosal layers, respectively. Whether these actually represent different diseases or simply different presentations of the same disease is not known. The prevalence of each subtype is unknown because of reporting and referral biases. For example, surgical series report a predominance of muscularis disease with obstruction, whereas medical series primarily describe patients with mucosal involvement. Serosal disease is associated with eosinophilic ascites, but it is not known whether this reflects isolated serosal involvement or simply intense transmural eosinophilic inflammation.

In addition to the common presentations noted above, EGE can present with a variety of unusual manifestations. Patients may have gastric ulcer disease as a feature of their EGE 9 . Typically these ulcers do not respond well to PPI therapy, but do respond to either topical or systemic corticosteroids. There is a case report of ulcer disease responding to an elemental $\operatorname{diet}^{10}$.

In contrast to EoE, stricture formation is not a common feature of EGE. That said, a subset of perhaps 5-10\% of EGE patients do have clinically significant strictures at some point. Such cases will typically present as an acute bowel obstruction with nausea, vomiting, crampy abdominal pain and bloating ${ }^{11}$. Such bowel obstructions appear to be a combination of both mechanical obstruction due to structuring as well as functional obstruction due to inflammation, edema, and decreased GI motility. Most of the time these obstructions are reversible with corticosteroid treatment, suggesting that in many cases there is a functional component that can be reversed with treatment ${ }^{12}$. As such, clinically stable EGE patients presenting with bowel obstruction should generally first be treated with parenteral corticosteroid therapy, such as methyl prednisolone $1-2 \mathrm{mg} / \mathrm{kg} /$ day and carefully observed. 
EGE can occasionally involve the hepatobiliary tree. Pancreatitis is the best described hepatobiliary complication of EGE ${ }^{13}$. It is not clear if this is due to eosinophilic infiltration of the pancreas, gall bladder or hepatobiliary ducts, or is due to a secondary cause. Additionally, EGE can rarely present with eosinophilic cholangitis ${ }^{14,15}$.

\section{Pathogenesis of EGE}

The many similarities between EGE and EoE suggest they share a common pathogenesis. Shared features include tissue eosinophilic inflammation, coexisting allergic disease, peripheral eosinophilia, and polysensitization to food allergens. The most compelling clinical feature of EoE, is its responsiveness to elemental and highly restricted diets ${ }^{16,17}$. Several case series suggest that at least a subpopulation of EGE are also responsive to elemental and 6 food elimination diets ${ }^{18,19}$. These findings underscore the concept that both EoE and EGE are food allergen driven eosinophilic inflammatory bowel diseases.

In an early report, Jaffe and colleagues noted increased IL-5 expression in PBMC from EGE patients ${ }^{20}$. Consistent with in vivo activation by food allergen, this IL-5 message was constitutively produced by CD4 T cells in the PBMC. A role for IL-5 in driving the peripheral eosinophilia has been further established by the use of therapeutic monoclonal anti-IL-5, which potently decreases peripheral eosinophils in $\mathrm{EGE}^{21}$.

In our own work, we examined food allergen specific CD4 T cell response in EGE, peanut allergic, and healthy control subjects and found differing $\mathrm{Th} 2$ responses in these eosinophilic vs. anaphylactic forms of food allergy ${ }^{22}$. Notably, EGE is uniquely associated with an IL-5+ Th2 (IL-5+, IL-13+) response to foods; conversely, in peanut allergy, the Th2 response is almost entirely IL-5- (IL-5-, IL-13+). This IL-5+ Th2 response is highly correlated to peripheral blood eosinophil count, further establishing a link between this Th2 subpopulation and eosinophilia. In recent work we have further characterized these IL-5+ Th2 cells as highly differentiated $\mathrm{Th} 2$ cells that require multiple rounds of antigen exposure to attain the IL-5+ Th2 phenotype ${ }^{23}$. These data suggest that in EGE these pathogenic proeosinophilic IL-5+ Th2 cells are the product of multiple rounds of food allergen stimulation in vivo.

EGE and EoE are defined by their respective sites of clinical disease. Despite these differences, their similarities far outweigh the differences and suggest the pathogenesis of these two forms of EGID have common mechanisms. The differences in site of disease may be due to local effects that may favor esophageal vs. gastric eosinophilic inflammation. For example, EoE may be influenced by high concentrations of swallowed aeroallergen impacting the esophagus, or by gastroesophageal reflux. Alternatively, there may be specific homing signals or receptors that favor esophageal vs. gastric homing.

\section{Diagnosis of EGE}

Unlike EoE, there are no consensus guidelines for the diagnosis of EGE. The diagnosis is based on typical symptoms coupled with increased gastric or intestinal eosinophils, in the absence of other potential causes of GI eosinophilia. There is no consensus on the requisite number of eosinophils needed for the diagnosis. As detailed in the accompanying chapter 
Histopathologic Features, based on studies of healthy controls, peak eosinophil counts of 30 eos/hpf in the stomach and 50 eos/hpf, in the duodenum have been proposed for the diagnosis of eosinophilic gastritis and duodenitis, respectively ${ }^{24,25}$. Additional features, such as epithelial eosinophils, intraglandular eosinophils, and eosinophils in the muscularis, also weight towards the diagnosis of EGE. The intensity of eosinophilic inflammation is quite variable within an affected organ and can reach almost confluent density in some cases (Figure 1). Accordingly, endoscopic biopsies should be obtained from 5-6 sites per affected organ, in a similar manner to the consensus procedures in $\mathrm{EoE}^{26}$.

Given the association of EGE with allergic disease, in clinical studies of "allergic" EGE, we have used additional criteria, such as IgE sensitization to multiple food allergens and peripheral blood eosinophilia ${ }^{22}$. These added criteria identify a subpopulation of EGE patients with more homogeneous immunological findings and add to the specificity of diagnosis. However, such criteria should not be used as an absolute requirement for the diagnosis, because there is a large fraction of EGE patient lacking these.

The gross endoscopic findings are often normal. Gastritis or ulcers may be present. The one observation that is relatively typical for EG is the presence of pseudopolyps ${ }^{10,27}$. These sessile lesions are not true polyps in that they are largely composed of dense collagen deposits with epithelium heaped on top. As such, they do not represent true polyps and do not contain hyperplastic glandular or epithelial components. Gastric pseudopolyps are the most common presentation (Fig 2) and may occur is as many as 25\% of EGE subjects (Prussin, personal observation). Small bowel pseudopolyps may occur, but are less frequent (Fig 3).

\section{Esophageal eosinophilia in patients with EGE}

As noted above EoE and EGE share many clinical and pathogenic features. Thus, it is not surprising that a subgroup of patients have widespread EGID that includes their esophagus, stomach and small bowel. Additionally, upon EGD, some patients with EoE will have increased numbers of gastric or duodenal eosinophils, of unclear significance. Gupta and colleagues found that $12 \%$ of pediatric EoE subjects had elevated gastric eosinophils, which they defined as $>10$ eos/hpf. They noted no significant clinical differences between EoE patients with or without incidental gastric eosinophilia ${ }^{28}$. Conversely, in our adult eosinophilic gastroenteritis clinic, we have noted $\approx 25 \%$ of EGE patients have $\geq 15 \mathrm{eos} / \mathrm{hpf}$ in their esophagus (Prussin, personal observation). Notably, only about half of those EGE patients with increased esophageal eosinophils have dysphagia. This dysphagia often responds to swallowed topical corticosteroids.

\section{Eosinophilia in patients with EGID}

Patients with EGID frequently present with elevated blood eosinophilia. This is particularly notable in EGE, in which a majority of patients present with blood eosinophilia ${ }^{3,6}$. This can often be a source of concern as eosinophil counts are often elevated into the several thousand range. Indeed, many of the EGE patients in our clinic were initially referred for hypereosinophilic syndrome (HES), but after careful work-up it was apparent that despite very high blood eosinophils counts, their disease was limited to the GI tract and did not 
involve other end organs. The absolute eosinophil count (AEC), measured in eos/mm3 or eos/microL, and not the percentage of eosinophils on the differential is the metric used to follow eosinophilia.

Recently proposed diagnostic criteria for HES define it as: blood eosinophilia of greater than $1500 / \mathrm{mm} 3$ on at least 2 occasions or evidence of prominent tissue eosinophilia associated with symptoms and marked blood eosinophilia ${ }^{29}$. This definition further excludes "secondary causes of eosinophilia, such as parasitic or viral infections, allergic diseases, drug-induced or chemical-induced eosinophilia, hypoadrenalism, and neoplasms". Given that EGID are clearly allergic in etiology, according to the new definition, EGID should not be considered a form of HES.

HES usually affects multiple organ systems, including the skin, lungs, GI tract, neurological system and heart ${ }^{30}$. Indeed, despite the frequency of high-grade eosinophilia in EGID, there is a conspicuous lack of reports in the literature of patients with stable allergic EGID of years duration at some later point transforming into an HES-like picture with skin ulcers, lung, heart, or neurological involvement. That said, the GI tract is one of the more commonly affected organ systems in HES and clearly there are rare patients who clinically present as an overlap between HES and EGID.

To address potential HES in EGID patients with AEC >1500 eos/mm2, we perform the following workup at initial presentation:

1. Drug history to examine for drug induced eosinophilia

2. Travel history

3. Stool for ova and parasites

4. Strongyloides stercoralis serology

5. T cell receptor clonality studies (to examine for a clonal $\mathrm{T}$ cell population involved in lymphocytic HES)

6. FIP1-like 1/platelet-derived growth factor receptor alpha fusion gene studies (to examine for the most common cause of myeloid HES)

7. Cardiac echo (to rule out endomyocardial fibrosis)

In addition to HES, eosinophilic granulomatosis with polyangiitis (EGPA, formally known as Churg-Strauss syndrome) shares many features with EGID and can present with GI symptoms. GI symptoms associated with EGPA can include dysphagia, abdominal pain, vomiting, anorexia, and bloody diarrhea. EGPA should be considered in the differential in EGID patients with marked asthma, pulmonary symptoms, or nasal polyps. The lung disease in EGPA characteristically has migratory infiltrates seen on chest radiograph. Serum antineutrophil cytoplasmic antibodies should be examined. Ultimately, the diagnosis is best made by the demonstration of eosinophil-rich necrotizing granulomatous vasculitis in the lungs or other site of disease. 


\section{Management of EGE}

Because of the low prevalence of EGE, there are no placebo controlled clinical trials examining therapeutic approaches. Although some patients may be optimally managed by the approaches noted below, most patients do not achieve complete remission of their EGE symptoms. As such, there is a great need for new approaches to the treatment of EGE.

Newly diagnosed patients are almost always responsive to systemic corticosteroid therapy. In patients who do not respond to corticosteroids, alternative diagnoses should be considered $^{3}$. Doses of prednisone of $0.5-1 \mathrm{mg} / \mathrm{kg}$ typically induce a dramatic clinical improvement in 2-14 days. As such, short-term treatment with systemic corticosteroids is an excellent means to induce clinical remission.

Because of the long-term side effects of systemic corticosteroids, topical corticosteroids have been used in a similar manner to those in EoE. Early practitioners used oral beclomethasone, often diluted in corn oil. A major limitation of beclomethasone is its oral bioavailability of approximately $30-40 \%{ }^{31}$. Given that beclomethasone has far great oral bioavailability than other topical corticosteroids, it should not be used in the contemporary treatment of EGE.

In contrast to beclomethasone, budesonide has an oral bioavailability of about $10 \%^{32}$. Similar to its use in EoE, budesonide has been used for the treatment of EGE ${ }^{12,33-35}$. Unfortunately, all publications are single case reports and are subject to reporting bias.

In the United States and European Union, the only commercial formulation of budesonide available in sufficient quantity to treat EGE is the controlled ileal release capsule preparation (Entocort EC). The controlled ileal release capsule provides optimal delivery to the terminal ileum, but provides minimal gastric delivery. Because most EGE patients have predominant gastric or duodenal disease, we routinely use a solubilized modification of these capsules to target topical activity to the upper GI tract. Such GI preparations of budesonide are not approved for use in pediatric patients, as their safety and efficacy of has not been established in this population. Accordingly, any off-label use in pediatric patients should only be done after careful consideration of the risks, benefits and alternatives.

For most symptomatic EGE patients we initiate therapy using prednisone at $0.4-0.8 \mathrm{mg} / \mathrm{kg}$ each morning to induce symptomatic remission. Simultaneously, solubilized budesonide is begun at $9 \mathrm{mg}$ PO daily, taken at bedtime on an empty stomach. Patients are advised to open the budesonide capsules, crush the contents in a mortar and pestle, and dissolve it in 15-30 $\mathrm{mL}$ of water and juice. In this manner there is minimal additional dilution of the drug and its upper GI dwell time is maximized, thus maximizing topical activity. Once clinical symptoms are controlled, prednisone is tapered over the next 2 or more weeks. One-two months after the prednisone has been stopped, the budesonide dose is slowly tapered over an additional 2-4 months to the minimum required dose. For patients with substantial protein losing enteropathy, in which jejunal or ileal disease is suspected, the intact controlled ileal capsule may be used. 
Most adult patients get improvement in symptoms on $9 \mathrm{mg} /$ day of budesonide. However, in many patients there are substantial systemic effects at that dose. A budesonide dose of 3-6 $\mathrm{mg}$ /day long-term is preferable. Ultimately, corticosteroid therapy in EGE is an imperfect compromise between a dose that yields a tolerable level of systemic side effects and only partial treatment of symptoms. That said, at the present time there are few other feasible medical treatment options for most patients.

Fluticasone is a fluorinated corticosteroid that is widely used in eosinophilic esophagitis and is notable for having an exceedingly low oral bioavailability of $\leq \%^{36}$. This makes it potentially an ideal drug for topical use in EGE. Unfortunately, fluticasone is only available in pulmonary and nasal inhalers, neither of which contain sufficient drug to treat EGE. Intriguingly, among pediatric EoE subjects with elevated gastric eosinophils who were treated with swallowed fluticasone, there was a significant decrease in gastric eosinophils ${ }^{28}$. This suggests that, particularly for gastric predominant EGE, higher doses of fluticasone are worthy of further study.

As in EoE, dietary therapy is an effective treatment in a large fraction of EGE patients. Chehade examined a cohort of 6 pediatric patients with allergic EGE and protein losing enteropathy ${ }^{19}$. All subjects responded to the elemental diet with a resolution of their clinical symptoms, hypoalbuminemia, and anemia in less than 4 weeks. When these same patients were managed with eliminations diet there was only limited success. Gonsalves published an abstract examining dietary therapy of 9 adults with $\mathrm{EGE}^{18}$. Two subjects were treated with elemental diet and both had resolution of both symptoms as well as tissue and blood eosinophilia. Seven subjects were treated with a 6-food elimination diet, of these, 4 of the 7 had resolution of both symptoms as well as tissue and blood eosinophilia. In sum, these data suggest that dietary approaches are generally effective, particularly in allergic EGE and should be given greater consideration.

Because EGE is an allergic disease and is associated with $\operatorname{IgE}$ sensitization to multiple foods, an open label clinical trial of the anti-IgE therapeutic monoclonal antibody was undertaken $^{37}$. As expected, omalizumab effectively suppressed free IgE and IgE-dependent basophil responses. Although the primary endpoint of peripheral blood eosinophil count was significantly decreased during the 4-month trial, tissue eosinophilia and symptoms were only modestly affected. Coupled with similar negative reports of omalizumab in EoE, these data suggest that IgE blockade is not an effective treatment for EGID ${ }^{38-40}$.

Given the central role of IL-5 in eosinophil biology and the abundance of evidence that IL-5 plays a major role in EGID, therapeutic monoclonal antibodies against IL-5 have been employed in EGID ${ }^{41}$. To this end, an open label clinical trial of the anti-IL-5 therapeutic monoclonal antibody reslizumab (SCH55700) was undertaken in 4 subjects with EGE $^{21}$. Reslizumab suppressed blood eosinophilia in a significant manner. However, tissue eosinophilia was only modestly suppressed and EGE symptoms were minimally affected. Additional work is needed to better define the efficacy of such approaches. The eosinophil depleting anti-IL-5 receptor therapeutic monoclonal antibody benralizumab may allow this question to be addressed in a more substantial manner in the future ${ }^{42}$. 
A variety of other drugs have been used in treat EGE and have been published in the literature, typically as case reports. Despite these generally positive reports, there are few controlled studies, nor is there a consensus among EGID specialists on the value of these drugs. Given the potential for reporting bias and non-reporting of negative data, until more definitive studies are done, these findings have to be considered with some skepticism.

In a number of case reports montekukast has been described as an effective treatment for EGE in ${ }^{43}, 44$. Furthermore, a retrospective case series of pediatric EGID patients including both EGE and EoE, montelukast improved EGID symptoms ${ }^{45}$. In contrast another report noted no benefit to treatment ${ }^{46}$. In a prospective study, $11 \mathrm{EoE}$ subjects were induced into remission using swallowed fluticasone and then switched to montelukast for 3 months ${ }^{47}$. Montelukast did not maintain either symptomatic or histological remission in this setting.

A variety of case reports have suggested sodium cromolyn is an effective treatment for $\mathrm{EGE}^{48-50}$. In contrast, another report notes its use was unsuccessful ${ }^{46}$. A clinical series of 14 EoE subjects treated with cromolyn at the University of Pennsylvania had no clinical or histological improvement in their disease $\mathrm{e}^{51}$.

Ketotifen is an $\mathrm{H} 1$ antihistamine that also has "mast cell stabilizing" activity. In the United States it is only available in eye drops, but is available as a systemic drug in Canada and the European Union. An early clinical trial in EGE indicated efficacy and several positive case reports have been published through the years ${ }^{52,53}$. Suplatast is an anti-Th2 drug that inhibits the expression of Th2 cytokines, such as IL-5. Suplatast is not available in the United States and European Union. Successful treatment of EGE with suplatast has been described in two single patient case reports, but the generalizability of these findings remains unclear ${ }^{54,55}$.

\section{Summary}

EGE is a subset of EGID characterized by intense eosinophilic infiltration of the stomach and small bowel. Although the pathogenesis of EGE shares many features of EoE, the differing localization of eosinophilic inflammation in EGE results in a different constellation of disease. Corticosteroids remain the mainstay of treatment.

Unfortunately, in many patients low dose topical corticosteroid therapy is not sufficiently effective to provide complete symptom relief. Thus, corticosteroid therapy in EGE is an imperfect compromise between a dose that yields a tolerable level of systemic side effects and only partial treatment of symptoms. Because EGE is a rare disease, relatively few clinical studies have been performed. As such, there is a large unmet need for new clinical therapeutic strategies.

\section{References}

1. Spergel JM, Book WM, Mays E, et al. Variation in prevalence, diagnostic criteria, and initial management options for eosinophilic gastrointestinal diseases in the United States. Journal of pediatric gastroenterology and nutrition. 2011; 52(3):300-306. [PubMed: 21057327]

2. Naylor AR. Eosinophilic gastroenteritis. Scottish medical journal. 1990; 35(6):163-165. [PubMed: 2077646] 
3. Talley NJ, Shorter RG, Phillips SF, et al. Eosinophilic gastroenteritis: a clinicopathological study of patients with disease of the mucosa, muscle layer, and subserosal tissues. Gut. 1990; 31(1):54-58. [PubMed: 2318432]

4. Kelly KJ. Eosinophilic gastroenteritis. Journal of pediatric gastroenterology and nutrition. 2000; 30(Suppl):S28-S35. [PubMed: 10634296]

5. Khan S. Eosinophilic gastroenteritis. Best practice \& research Clinical gastroenterology. 2005; 19(2):177-198. [PubMed: 15833687]

6. Zhang L, Duan L, Ding S, et al. Eosinophilic gastroenteritis: clinical manifestations and morphological characteristics, a retrospective study of 42 patients. Scandinavian journal of gastroenterology. 2011; 46(9):1074-1080. [PubMed: 21623674]

7. Khan F, Chaudhry MA, Nusrat S, et al. Constipation--another manifestation of eosinophilic gastroenteritis. The Journal of the Oklahoma State Medical Association. 2012; 105(4-5):134-136. [PubMed: 22803505]

8. Chang JY, Choung RS, Lee RM, et al. A shift in the clinical spectrum of eosinophilic gastroenteritis toward the mucosal disease type. Clinical gastroenterology and hepatology : the official clinical practice journal of the American Gastroenterological Association. 2010; 8(8):669-675. quiz e688. [PubMed: 20451664]

9. Kristopaitis T, Neghme C, Yong SL, et al. Giant antral ulcer: a rare presentation of eosinophilic gastroenteritis--case report and review of the literature. The American journal of gastroenterology. 1997; 92(7):1205-1208. [PubMed: 9219801]

10. Chehade M, Sicherer SH, Magid MS, et al. Multiple exudative ulcers and pseudopolyps in allergic eosinophilic gastroenteritis that responded to dietary therapy. Journal of pediatric gastroenterology and nutrition. 2007; 45(3):354-357. [PubMed: 17873749]

11. Yun MY, Cho YU, Park IS, et al. Eosinophilic gastroenteritis presenting as small bowel obstruction: a case report and review of the literature. World journal of gastroenterology : WJG. 2007; 13(11):1758-1760. [PubMed: 17461485]

12. Elsing C, Placke J, Gross-Weege W. Budesonide for the treatment of obstructive eosinophilic jejunitis. Zeitschrift fur Gastroenterologie. 2007; 45(2):187-189. [PubMed: 17304405]

13. Lyngbaek S, Adamsen S, Aru A, et al. Recurrent acute pancreatitis due to eosinophilic gastroenteritis. Case report and literature review. JOP : Journal of the pancreas. 2006; 7(2):211217. [PubMed: 16525206]

14. Schoonbroodt D, Horsmans Y, Laka A, et al. Eosinophilic gastroenteritis presenting with colitis and cholangitis. Digestive diseases and sciences. 1995; 40(2):308-314. [PubMed: 7851195]

15. Nashed C, Sakpal SV, Shusharina V, et al. Eosinophilic cholangitis and cholangiopathy: a sheep in wolves clothing. HPB surgery : a world journal of hepatic, pancreatic and biliary surgery. 2010; 2010:906496.

16. Markowitz JE, Spergel JM, Ruchelli E, et al. Elemental diet is an effective treatment for eosinophilic esophagitis in children and adolescents. The American journal of gastroenterology. 2003; 98(4):777-782. [PubMed: 12738455]

17. Chehade M, Aceves SS. Food allergy and eosinophilic esophagitis. Current opinion in allergy and clinical immunology. 2010; 10(3):231-237. [PubMed: 20410819]

18. Gonsalves N, Doerfler B, Yang GY, et al. A Prospective Clinical Trial of Six Food Elimination Diet or Elemental Diet in the Treatment of Adults with Eosinophilic Gastroenteritis. Gastroenterology. 2009; 136(5):A280-A280.

19. Chehade M, Magid MS, Mofidi S, et al. Allergic eosinophilic gastroenteritis with protein-losing enteropathy: intestinal pathology, clinical course, and long-term follow-up. Journal of pediatric gastroenterology and nutrition. 2006; 42(5):516-521. [PubMed: 16707973]

20. Jaffe JS, James SP, Mullins GE, et al. Evidence for an abnormal profile of interleukin-4 (IL-4), IL-5, and gamma-interferon (gamma-IFN) in peripheral blood T cells from patients with allergic eosinophilic gastroenteritis. J Clin Immunol. 1994; 14(5):299-309. [PubMed: 7814459]

21. Kim YJ, Prussin C, Martin B, et al. Rebound eosinophilia after treatment of hypereosinophilic syndrome and eosinophilic gastroenteritis with monoclonal anti-IL-5 antibody SCH55700. The Journal of allergy and clinical immunology. 2004; 114(6):1449-1455. [PubMed: 15577851] 
22. Prussin C, Lee J, Foster B. Eosinophilic gastrointestinal disease and peanut allergy are alternatively associated with IL-5+ and IL-5(-) T(H)2 responses. The Journal of allergy and clinical immunology. 2009; 124(6):1326-1332. e1326. [PubMed: 20004787]

23. Upadhyaya B, Yin Y, Hill BJ, et al. Hierarchical IL-5 expression defines a subpopulation of highly differentiated human Th2 cells. Journal of immunology. 2011; 187(6):3111-3120.

24. Lwin T, Melton SD, Genta RM. Eosinophilic gastritis: histopathological characterization and quantification of the normal gastric eosinophil content. Modern pathology : an official journal of the United States and Canadian Academy of Pathology, Inc. 2011; 24(4):556-563.

25. DeBrosse CW, Case JW, Putnam PE, et al. Quantity and distribution of eosinophils in the gastrointestinal tract of children. Pediatric and developmental pathology : the official journal of the Society for Pediatric Pathology and the Paediatric Pathology Society. 2006; 9(3):210-218.

26. Liacouras CA, Furuta GT, Hirano I, et al. Eosinophilic esophagitis: updated consensus recommendations for children and adults. The Journal of allergy and clinical immunology. 2011; 128(1):3-20. e26; quiz 21-22. [PubMed: 21477849]

27. Jimenez-Rivera C, Ngan B, Jackson R, et al. Gastric pseudopolyps in eosinophilic gastroenteritis. Journal of pediatric gastroenterology and nutrition. 2005; 40(1):83-86. [PubMed: 15625432]

28. Ammoury RF, Rosenman MB, Roettcher D, et al. Incidental gastric eosinophils in patients with eosinophilic esophagitis: do they matter? Journal of pediatric gastroenterology and nutrition. 2010; 51(6):723-726. [PubMed: 20601904]

29. Simon HU, Rothenberg ME, Bochner BS, et al. Refining the definition of hypereosinophilic syndrome. The Journal of allergy and clinical immunology. 2010; 126(1):45-49. [PubMed: 20639008]

30. Ogbogu PU, Bochner BS, Butterfield JH, et al. Hypereosinophilic syndrome: a multicenter, retrospective analysis of clinical characteristics and response to therapy. The Journal of allergy and clinical immunology. 2009; 124(6):1319-1325. e1313. [PubMed: 19910029]

31. Daley-Yates PT, Price AC, Sisson JR, et al. Beclomethasone dipropionate: absolute bioavailability, pharmacokinetics and metabolism following intravenous, oral, intranasal and inhaled administration in man. British journal of clinical pharmacology. 2001; 51(5):400-409. [PubMed: 11421996]

32. Friend DR. Review article: issues in oral administration of locally acting glucocorticosteroids for treatment of inflammatory bowel disease. Alimentary pharmacology \& therapeutics. 1998; 12(7): 591-603. [PubMed: 9701522]

33. Tan AC, Kruimel JW, Naber TH. Eosinophilic gastroenteritis treated with non-enteric-coated budesonide tablets. European journal of gastroenterology \& hepatology. 2001; 13(4):425-427. [PubMed: 11338074]

34. Siewert E, Lammert F, Koppitz P, et al. Eosinophilic gastroenteritis with severe protein-losing enteropathy: successful treatment with budesonide. Digestive and liver disease : official journal of the Italian Society of Gastroenterology and the Italian Association for the Study of the Liver. 2006; 38(1):55-59.

35. Lombardi C, Salmi A, Passalacqua G. An adult case of eosinophilic pyloric stenosis maintained on remission with oral budesonide. European annals of allergy and clinical immunology. 2011; 43(1): 29-30. [PubMed: 21409859]

36. Hubner M, Hochhaus G, Derendorf H. Comparative pharmacology, bioavailability, pharmacokinetics, and pharmacodynamics of inhaled glucocorticosteroids. Immunology and allergy clinics of North America. 2005; 25(3):469-488. [PubMed: 16054538]

37. Foroughi S, Foster B, Kim N, et al. Anti-IgE treatment of eosinophil-associated gastrointestinal disorders. The Journal of allergy and clinical immunology. 2007; 120(3):594-601. [PubMed: 17765756]

38. Fang JC, Hilden K, Gleich GJ, et al. A Pilot Study of the Treatment of Eosinophilic Esophagitis With Omalizumab. Gastroenterology. 2011; 140(5):S235-S235.

39. Rocha R, Vitor AB, Trindade E, et al. Omalizumab in the treatment of eosinophilic esophagitis and food allergy. European journal of pediatrics. 2011; 170(11):1471-1474. [PubMed: 21809010]

40. Loizou D, Louis-Jacques O, Enav B, et al. Elucidating Mechanisms of Allergic Inflammation in Eosinophilic Esophagitis. J Allergy Clin Immun. 2013; 131(2):Ab184-Ab184. 
41. Wechsler ME, Fulkerson PC, Bochner BS, et al. Novel targeted therapies for eosinophilic disorders. J Allergy Clin Immun. 2012; 130(3):563-571. [PubMed: 22935585]

42. Molfino NA, Gossage D, Kolbeck R, et al. Molecular and clinical rationale for therapeutic targeting of interleukin-5 and its receptor. Clinical and experimental allergy : journal of the British Society for Allergy and Clinical Immunology. 2012; 42(5):712-737. [PubMed: 22092535]

43. Neustrom MR, Friesen C. Treatment of eosinophilic gastroenteritis with montelukast. The Journal of allergy and clinical immunology. 1999; 104(2 Pt 1):506. [PubMed: 10452782]

44. Schwartz DA, Pardi DS, Murray JA. Use of montelukast as steroid-sparing agent for recurrent eosinophilic gastroenteritis. Digestive diseases and sciences. 2001; 46(8):1787-1790. [PubMed: 11508684]

45. Vanderhoof JA, Young RJ, Hanner TL, et al. Montelukast: use in pediatric patients with eosinophilic gastrointestinal disease. Journal of pediatric gastroenterology and nutrition. 2003; 36(2):293-294. [PubMed: 12548071]

46. Daikh BE, Ryan CK, Schwartz RH. Montelukast reduces peripheral blood eosinophilia but not tissue eosinophilia or symptoms in a patient with eosinophilic gastroenteritis and esophageal stricture. Annals of allergy, asthma \& immunology : official publication of the American College of Allergy, Asthma, \& Immunology. 2003; 90(1):23-27.

47. Lucendo AJ, De Rezende LC, Jimenez-Contreras S, et al. Montelukast was inefficient in maintaining steroid-induced remission in adult eosinophilic esophagitis. Digestive diseases and sciences. 2011; 56(12):3551-3558. [PubMed: 21674173]

48. Van Dellen RG, Lewis JC. Oral administration of cromolyn in a patient with protein-losing enteropathy, food allergy, and eosinophilic gastroenteritis. Mayo Clinic proceedings. 1994; 69(5): 441-444. [PubMed: 8170195]

49. Perez-Millan A, Martin-Lorente JL, Lopez-Morante A, et al. Subserosal eosinophilic gastroenteritis treated efficaciously with sodium cromoglycate. Digestive diseases and sciences. 1997; 42(2):342-344. [PubMed: 9052516]

50. Suzuki J, Kawasaki Y, Nozawa R, et al. Oral disodium cromoglycate and ketotifen for a patient with eosinophilic gastroenteritis, food allergy and protein-losing enteropathy. Asian Pacific journal of allergy and immunology / launched by the Allergy and Immunology Society of Thailand. 2003; 21(3):193-197. [PubMed: 15032404]

51. Liacouras CA, Spergel JM, Ruchelli E, et al. Eosinophilic esophagitis: a 10-year experience in 381 children. Clinical gastroenterology and hepatology : the official clinical practice journal of the American Gastroenterological Association. 2005; 3(12):1198-1206. [PubMed: 16361045]

52. Melamed I, Feanny SJ, Sherman PM, et al. Benefit of ketotifen in patients with eosinophilic gastroenteritis. The American journal of medicine. 1991; 90(3):310-314. [PubMed: 2003512]

53. Freeman HJ. Longstanding eosinophilic gastroenteritis of more than 20 years. Canadian journal of gastroenterology = Journal canadien de gastroenterologie. 2009; 23(9):632-634. [PubMed: 19816628]

54. Shirai T, Hashimoto D, Suzuki K, et al. Successful treatment of eosinophilic gastroenteritis with suplatast tosilate. The Journal of allergy and clinical immunology. 2001; 107(5):924-925. [PubMed: 11344364]

55. Ishido K, Tanabe S, Higuchi K, et al. Eosinophilic gastroenteritis associated with giant folds. Digestive endoscopy : official journal of the Japan Gastroenterological Endoscopy Society. 2010; 22(4):312-315. [PubMed: 21175485] 


\section{Key Points}

1. Eosinophilic gastroenteritis (EGE) is diagnosed by the presence of gastrointestinal symptoms, biopsies showing predominant eosinophilic infiltration, and the absence of allergic, parasitic or other diseases that may cause eosinophilia.

2. EGE is a rare disease affecting approximately $22-28$ per 100,000 persons.

3. Because EGE may vary by both site of involvement (stomach, duodenum, jejunum) and the depth of involvement (mucosal, muscularis, or serosal disease), its manifestations are protean.

4. Dietary therapy is effective in allergic EGE

5. Systemic and topical corticosteroids are effective treatments for EGE, but are limited by long-term corticosteroid side effects. 


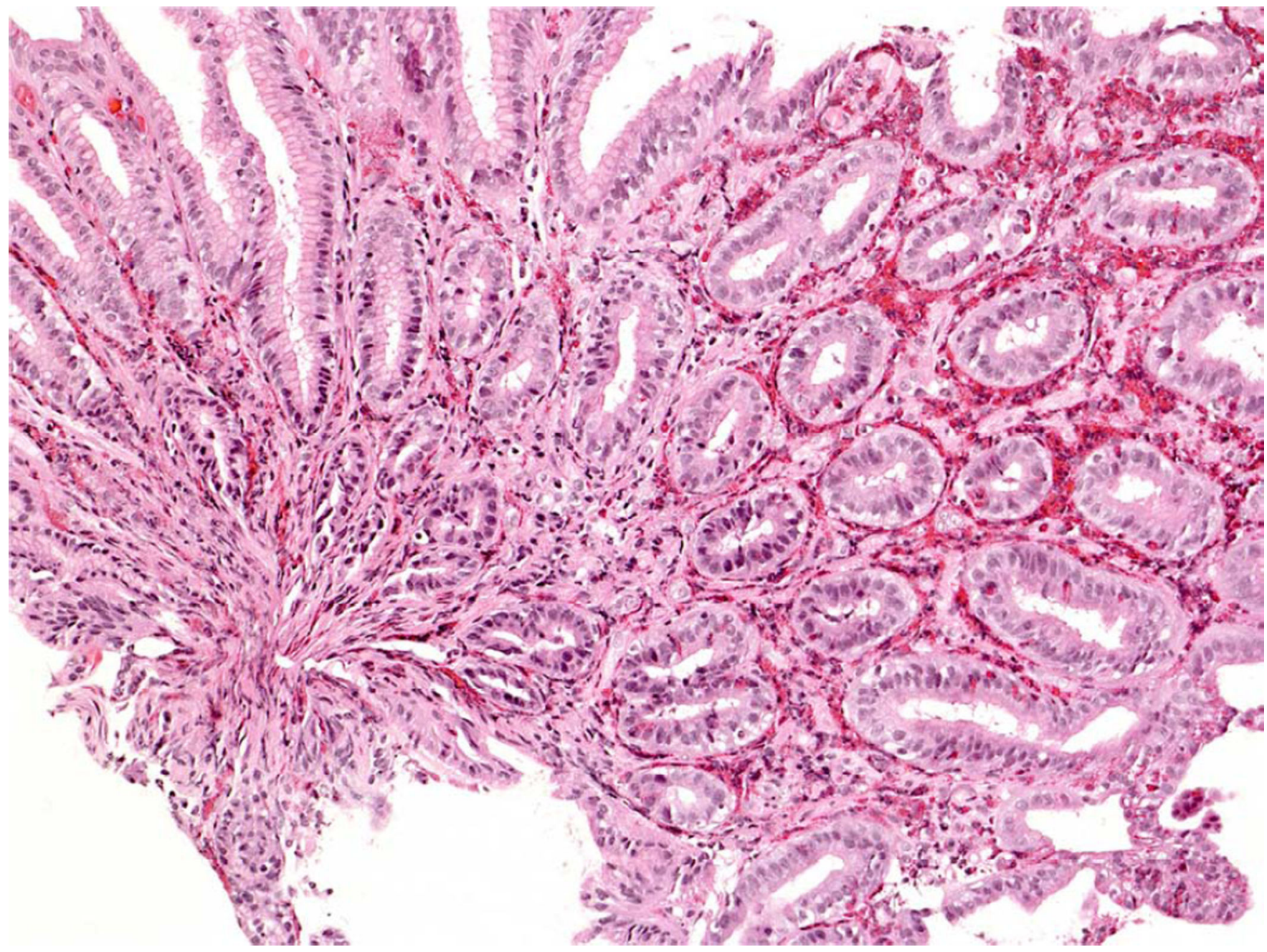

Figure 1.

Duodenal biopsy showing intense areas of eosinophilic inflammation. 


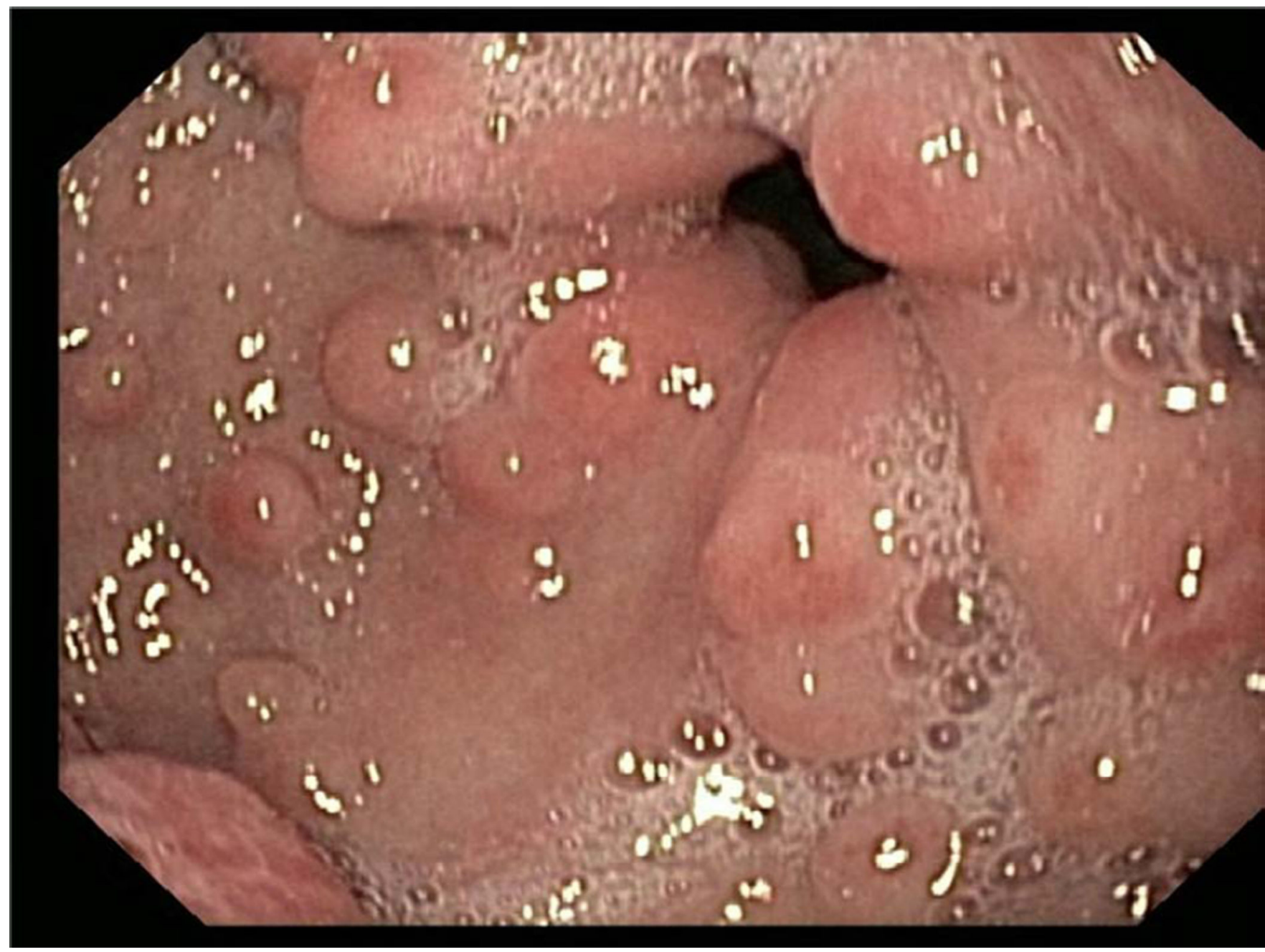

Figure 2.

Gastric pseudopolyps involving the gastric antrum and pyloris 


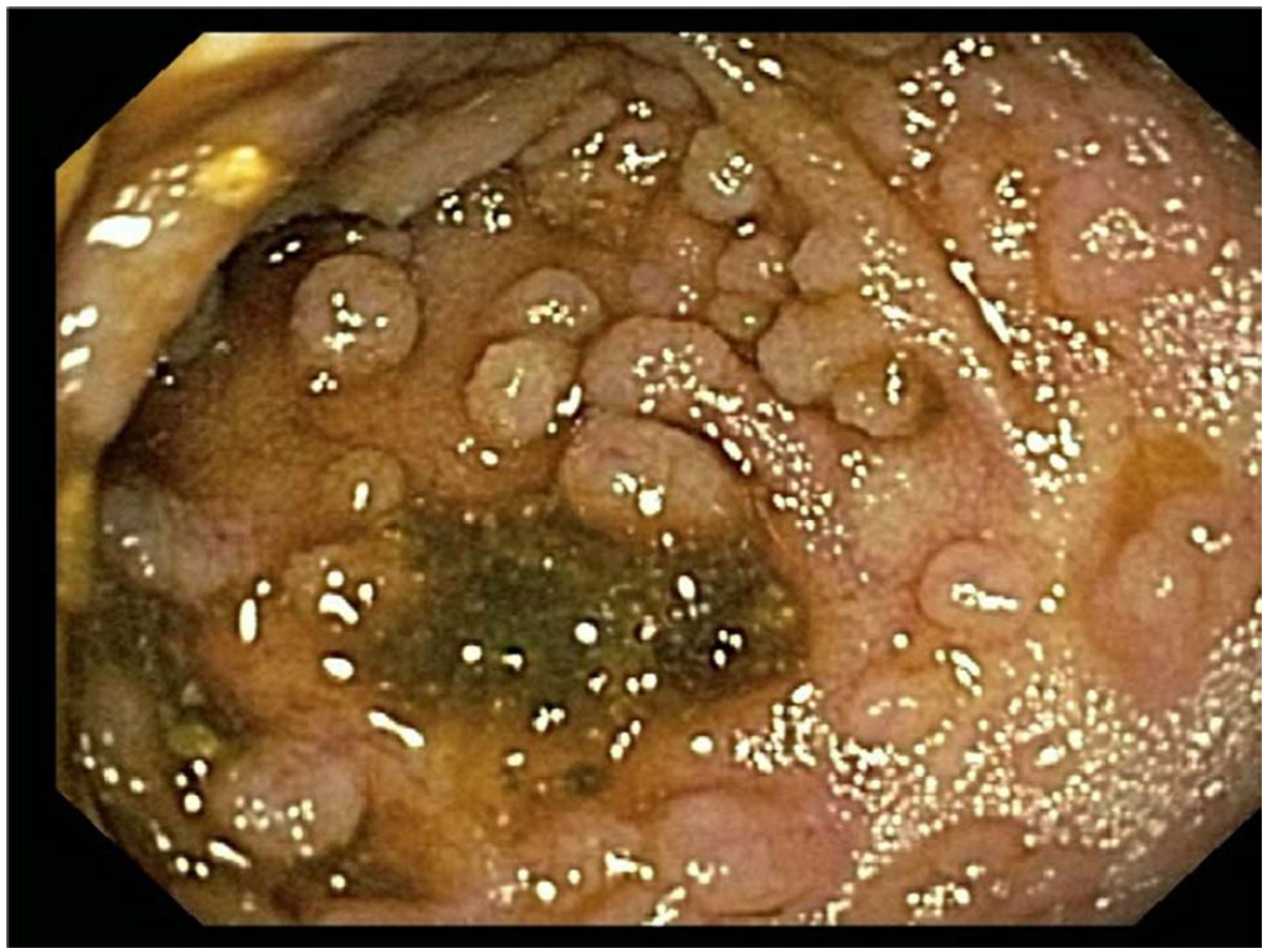

Figure 3.

Pseudopolyps within the terminal ileum. 